\title{
Goniospectrophotometric characterization of coatings with diffraction pigments
}

\author{
A. Ferrero ${ }^{1, *}$, B. Bernad ${ }^{1}$, J. Campos $^{1}$, E. Perales ${ }^{2}$, J. L. Velázquez ${ }^{1}$, and F. M. \\ MARTÍNEZ-VERDÚ ${ }^{2}$ \\ ${ }^{1}$ Instituto de Óptica, Consejo Superior de Investigaciones Científicas (CSIC), Madrid, Spain \\ ${ }^{2}$ Department of Optics, Pharmacology and Anatomy, University of Alicante, Carretera de San Vicente del Raspeig s/n 03690, Alicante, Spain
}

Compiled May 31, 2016

\begin{abstract}
Coatings with diffraction pigments present high iridescence, which needs to be characterized to describe their appearance. The spectral Bidirectional Reflectance Distribution Function (BRDF) of six coatings with SpectraFlair diffraction pigments were measured using the robot-arm-based goniospectrophotometerer GEFE, designed and developed at CSIC. Principal Components Analysis (PCA) has been applied to study the coatings' BRDF data. From data evaluation and based in theoretical considerations, we propose a relevant geometric factor to study the spectral reflectance and color gamut variation of coatings with diffraction pigments. At fixed values of this geometrical variable, the spectral BRDF component due to diffraction is almost constant. Commercially-available portable goniospectrophotometers, extensively used in several industries (automotive and others), have to be provided with more aspecular measurement angles to characterize goniochromatic coatings based on diffraction pigments.
\end{abstract}

OCIS codes: (290.1483) BSDF, BRDF, and BTDF, (050.1940) Diffraction, (030.5630) Radiometry, (120.5820) Scattering measurements.

http://dx.doi.org/10.1364/ao.XX.XXXXXX

\section{INTRODUCTION}

The complex reflectance properties of coatings with effect pigments result in quite strange and eye-catching color sensation for humans. As a consequence of their appealing appearance, they have become very popular in the automotive industry [1], and they are also widely used in other markets, such as the cosmetics industry. The complexity of their reflectance also allows them to be used for more functional purposes, as anticounterfeiting.

The color of effect pigments is caused by anisotropic optical processes like low-order scattering, interference, or diffraction. With regard to these processes, they can be basically divided into three types:

1. Metallic pigments. Their optical properties are described essentially by geometrical optics. They consist of a metal or an alloy of metal. Their metallic effect is mainly due to the directional reflection at the surface of the flake pigments.

2. Interference pigments. Unlike metallic pigments, their optical properties are described by wave optics. They consist of two or more layers with a high refractive index difference, which produces interference of the light waves. This interference is only observed at specular and near specular collection directions. Due to this interference, their color depends on the incidence angle on the pigment.

3. Diffraction pigments. Like interference pigments, their optical properties are described by wave optics. They have a grating structure which deflects the incoming light. Therefore, unlike metallic or interference pigments, their effect is not only observed at specular and near specular collection directions, but also at every direction. Since the deflection depends on the wavelength, the color at a given collection direction depends on the deflection angle.

Because of their optical properties, interference and diffraction pigments present iridescence, a very strong hue change for different pairs of irradiation and collection directions. Some insects, as for instance members of some genera of Lepidoptera and Coleoptera [2], present iridescence in their wings, and this suggests that the hue change might provide rich information for them. However, the accurate characterization of this effect is still challenging and deserve attention to understand their functions in nature. Microspectrophotometric techniques have been recently developed to study the optical properties for these biological structures [3,4]. But unfortunately, although they allow high spatial resolution, these analyses still require assessment of the reflection in bidirectional terms, that is, for given irradiation and collection directions, which is mandatory if we want 
to understand the real effect of interference and diffraction pigments.

In the last years, IO-CSIC's group has been working to improve the measurement of the spectral Bidirectional Reflectance Distribution Function (BRDF) to metrological accuracy. The BRDF of a surface depicts how it scatters the optical radiation, being therefore fundamental to explain its appearance. Although it is defined as the derivative of the radiance in the collection direction with respect to the irradiance from the irradiation direction [5], it is usually expressed as the ratio between both quantities, which is valid in the domain where the derivative remains constant. To completely characterize the spectral BRDF it is necessary to provide spectral data for key geometries (a geometry is a pair of irradiation and collection directions), sufficient to obtain interpolated data at intermediate geometries with enough accuracy.

IO-CSIC developed the goniospectrophotometer GEFE [6, 7] with the objective of giving insight into the appearance of surfaces with complex reflectance. This instrument has been recently used to study coatings with interference pigments widely used in automotive industry, in order to provide with general key geometries for their characterization [8-10]. In this work, we take advantage of this previous experience in the study of coatings with interference pigments to propose a general methodology for the characterization of coatings with diffraction pigments. We hope that it may contribute to the design of commercial multi-angle spectrophotometers for controlling the appearance of these coatings, but also that it may provide ideas for research in other fields, as in the structural colors in biology.

Similarly to our study on coatings with interference pigments, we have measured the spectral BRDF for a large number of uniformly-distributed geometries and, by using principal components analysis (PCA) as mathematical tool and basic physical considerations, we have investigated the requirements to completely characterize the spectral BRDF of coatings with diffraction pigments, and therefore their complex appearances. Previous experimental studies on coatings with diffraction coatings have been recently published, showing the color travel of these coatings in $\mathrm{CIE} \mathrm{a}^{*}, \mathrm{~b}^{*}$-diagrams $[11,12]$. Interestingly, theoretical simulations can be found in literature [13], giving insight in the dependence of the spectral reflectance on the period of the diffractive grating and its amplitude.

\section{COATINGS WITH DIFFRACTION PIGMENTS}

Reflective diffraction pigments are of special interest in industrial color physics. They basically consist of a metal substrate with a grating of embossed parallel grooves. This substrate is coated by inorganic substances forming a so called diffraction pigment flake (see Fig. 1).

The spectral reflectance of diffraction pigments depends on the geometry, since for every pair of irradiation and collection directions the optical path difference $\Delta G$ introduced by the diffraction pigment is different. When the direction of the grooves is perpendicular to the incidence plane, $\Delta G$ can be expressed as [11]:

$$
\Delta G=d\left(\sin \alpha_{\mathrm{i}}+\sin \alpha_{\mathrm{r}}\right)
$$

where $d$ is the grating period, $\alpha_{\mathrm{i}}$ is the angle of incidence on the pigment flake and $\alpha_{\mathrm{r}}$ is the angle of the collection direction, both in the incidence plane (Fig. 2). As convention, $\alpha_{\mathrm{i}}$ is always positive and $\alpha_{\mathrm{r}}$ is negative if the collection direction is contained in the same half-plane than the specular direction.
When the direction of the grooves is not perpendicular to the incidence plane, the diffraction is not observed anymore within the incidence plane, but in a plane defined by the specular direction (zeroth-order diffraction) and the direction perpendicular to the grooves within the flake surface. We will show that in this case the expression of $\Delta G$ must be modified to include the azimuth spherical coordinate of the collection direction $\phi_{\mathrm{r}}$.

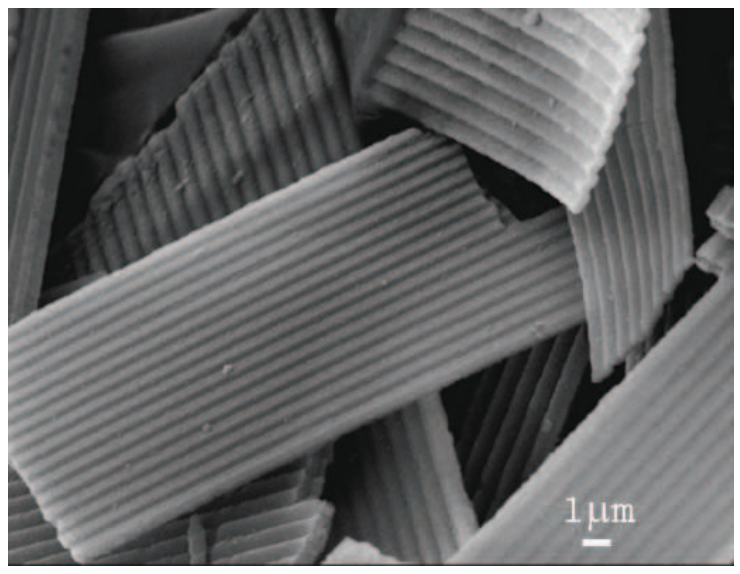

Fig. 1. Picture of a diffraction pigment (source: Viavi Solutions Inc, Milpitas, CA, USA).

In the effect coatings, the diffraction pigments are embedded in a transparent medium or binder with a refractive index $n$. Within this medium, the flake pigments are randomly oriented and, therefore, the observed diffraction corresponds to the superposition of diffraction patterns produced by the different orientations of the grooves. In addition, the flake pigments are not always perfectly parallel to the surface of the coatings, but they can be tilted an angle $\theta_{\text {flake }}$ (Fig. 2), which should cause a slight blurring in the diffraction pattern. In order to describe the diffraction on these coatings, we will consider this not perfect horizontality as a second-order effect, which means that we will assume that the average value of $\theta_{\text {flake }}$ is small and that this effect on the spectral BRDF is negligible.

Reflection geometric scheme on effect coatings is shown in Fig. 2. An irradiation/collection geometry is characterized by the spherical coordinates of their directions. $\theta_{\mathrm{i}}$ and $\theta_{\mathrm{r}}$ are the polar angles of irradiation and collection directions, respectively. For the sake of clarity, azimuth angles of irradiation and collection directions $\left(\phi_{\mathrm{i}}\right.$ and $\left.\phi_{\mathrm{r}}\right)$ are not shown in this figure. We note that here always $\phi_{\mathrm{i}}=0^{\circ}$, and, therefore, the value of $\phi_{\mathrm{r}}$ represents the difference between the azimuth angles of the irradiation and collection directions.

Within the incidence plane, the path difference $\Delta G_{\text {in }}$ for a given irradiation/collection geometry is calculated as in Eq. 1 . If we assume that the spectral BRDF is independent of the distribution of $\theta_{\text {flake }}\left(\theta_{\text {flake }} \approx 0^{\circ}\right)$, then we can write:

$$
\Delta G_{\text {in }} \approx d\left(\sin \theta_{\mathrm{i}}^{\prime}+\sin \theta_{\mathrm{r}}^{\prime}\right)
$$

and, according to the Snell's law:

$$
\begin{aligned}
& \sin \theta_{\mathrm{i}}=n \sin \theta_{\mathrm{i}}^{\prime} \\
& \sin \theta_{\mathrm{r}}=n \sin \theta_{\mathrm{r}}^{\prime}
\end{aligned}
$$

we can rewrite Eq. 2 as:

$$
\Delta G_{\text {in }} \approx \frac{d}{n}\left(\sin \theta_{\mathrm{i}}+\sin \theta_{\mathrm{r}}\right)
$$




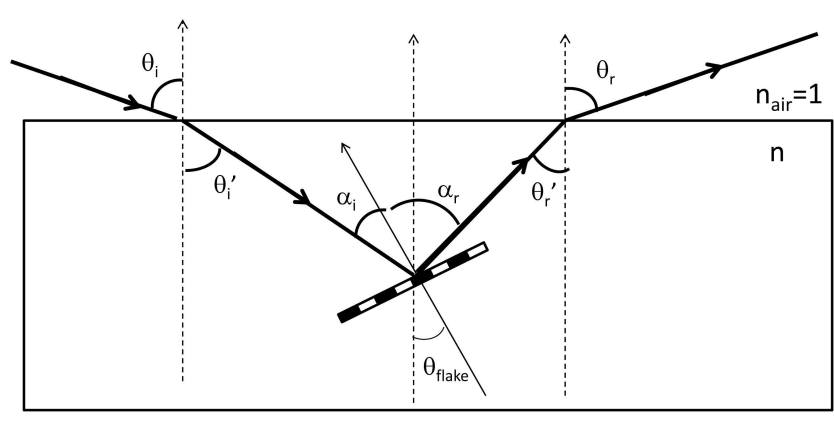

Fig. 2. Geometric scheme for reflection on coatings with diffraction pigments.

where, just for this equation, the same convention as for $\alpha_{\mathrm{r}}$ is used for the sign of $\theta_{\mathrm{r}}$.

We need to generalize this equation to include diffraction out of the incidence plane. The diffraction observed out of the incidence plane is produced by the diffraction pigments whose grooves are not perpendicular to this plane. In any case, the path difference introduced by a diffractive grating at a given geometry can be expressed as:

$$
\Delta G=G_{2}-G_{1}=d \hat{\mathbf{u}}_{\mathrm{d}} \cdot \hat{\mathbf{u}}_{\mathrm{r}}-d \hat{\mathbf{u}}_{\mathrm{d}} \cdot \hat{\mathbf{u}}_{0}
$$

where $G_{1}$ and $G_{2}$ are the additional paths of rays 1 and rays 2 (both incident from the direction defined by the unit vector $\hat{\mathbf{u}}_{\mathrm{i}}$ ), which are calculated as projections (scalar products) of $\hat{\mathbf{u}}_{\mathrm{d}}$ on $\hat{\mathbf{u}}_{\mathrm{r}}$ and $\hat{\mathbf{u}}_{0}$ (see Fig. 3), which are the unit vectors representing the direction perpendicular to the grooves within the pigment's plane, the collection direction and the zeroth-order diffraction direction, respectively.

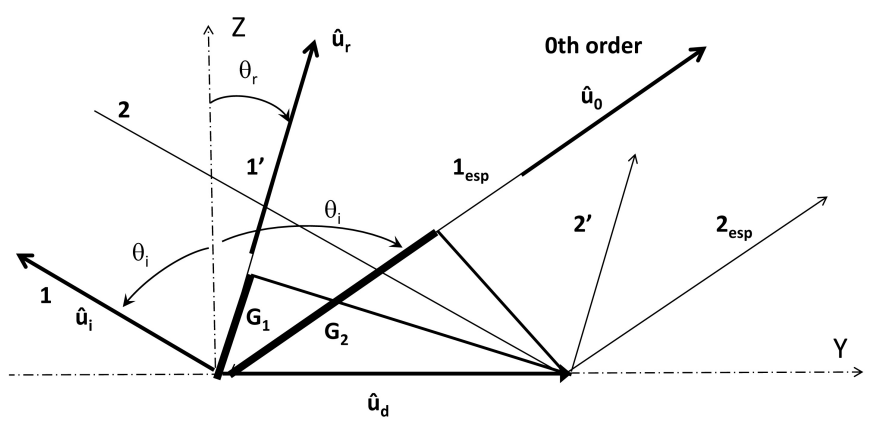

(a) Two dimensional diagram.

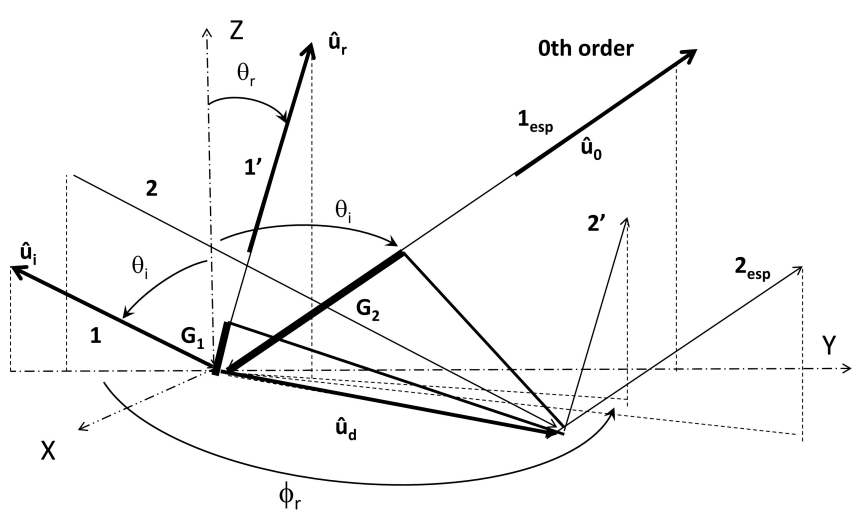

(b) Three dimensional diagram.

Fig. 3. Geometric scheme for calculating the path difference introduced by a diffractive grating. Two parallel rays ( 1 and 2 ) reflected ( 1 ' and $\left.2^{\prime}\right)$ on two separate points of a surface have different optical paths $\left(G_{1}\right.$ and $\left.G_{2}\right)$.

Except for the case of zeroth-order diffraction, the diffracted flux at given irradiation and collection directions corresponds with only one orientation of the grooves. This orientation is given by the unit vector $\hat{\mathbf{u}}_{\mathrm{d}}$, which can be obtained for any diffracted flux at any geometry defined by $\left(\hat{\mathbf{u}}_{\mathrm{i}}, \hat{\mathbf{u}}_{\mathrm{r}}\right)$ by considering that diffraction is produced only in the plane defined by the zeroth-order direction $\left(\hat{\mathbf{u}}_{0}\right)$ and the direction perpendicular to the grooves $\left(\hat{\mathbf{u}}_{\mathrm{d}}\right)$. This plane (the diffraction plane hereafter) is defined by its normal vector $\mathbf{N}_{\text {dif }}$, calculated as the vector product between the zeroth order direction and the direction at which diffraction is observed:

$$
\mathbf{N}_{\text {dif }}=\hat{\mathbf{u}}_{0} \wedge \hat{\mathbf{u}}_{\mathrm{r}}
$$

Since $\hat{\mathbf{u}}_{\mathrm{d}}$ is included in the plane containing the diffractive grating $(X Y)$, its direction is given by the intersection between the diffraction plane, defined by its normal $\mathbf{N}_{\text {dif }}$, and the diffractive grating plane $(X Y)$, defined by its normal vector $\mathbf{N}_{X Y}=$ $(0,0,1)$. Then $\hat{\mathbf{u}}_{\mathrm{d}}$ can be calculated as the vector product:

$$
\hat{\mathbf{u}}_{\mathrm{d}}=\frac{\mathbf{N}_{\mathrm{dif}} \wedge \mathbf{N}_{\mathrm{XY}}}{\left\|\mathbf{N}_{\mathrm{dif}} \wedge \mathbf{N}_{\mathrm{XY}}\right\|}
$$

So, we can say that the diffraction observed out of the plane of incidence is produced by diffraction gratings whose orientations are defined by $\hat{\mathbf{u}}_{\mathrm{d}}$, which in turn can be exclusively calculated from the irradiation and collection (diffraction) directions 
$\left(\hat{\mathbf{u}}_{\mathrm{i}}, \hat{\mathbf{u}}_{\mathrm{r}}\right)$. By using Eqs. 6, 7 and 8:

$$
\Delta G=d\left[\frac{\left(\hat{\mathbf{u}}_{0} \wedge \hat{\mathbf{u}}_{\mathrm{r}}\right) \wedge \mathbf{N}_{\mathrm{XY}}}{\left\|\left(\hat{\mathbf{u}}_{0} \wedge \hat{\mathbf{u}}_{\mathrm{r}}\right) \wedge \mathbf{N}_{\mathrm{XY}}\right\|}\right] \cdot\left(\hat{\mathbf{u}}_{\mathrm{r}}-\hat{\mathbf{u}}_{0}\right)
$$

By arranging it to be explicitly expressed as a function of spherical coordinates, we obtain:

$$
\Delta G=d \Phi\left(\theta_{\mathrm{i}}, \theta_{\mathrm{r}}, \phi_{\mathrm{r}}\right)
$$

where $\Phi$ is a geometric factor defined as:

$$
\begin{array}{r}
\Phi\left(\theta_{\mathrm{i}}, \theta_{\mathrm{r}}, \phi_{\mathrm{r}}\right)= \\
\frac{\left(\sin \theta_{\mathrm{i}}+\sin \theta_{\mathrm{r}} \cos \phi_{\mathrm{r}}\right) \tan \theta_{\mathrm{i}}+\left(\sin \theta_{\mathrm{i}} \cos \phi_{\mathrm{r}}+\sin \theta_{\mathrm{r}}\right) \tan \theta_{\mathrm{r}}}{\sqrt{\tan ^{2} \theta_{\mathrm{i}}+\tan ^{2} \theta_{\mathrm{r}}+2 \tan \theta_{\mathrm{i}} \tan \theta_{\mathrm{r}} \cos \phi_{\mathrm{r}}}}
\end{array}
$$

Thus, Eqs. 10 and 11 represent the generalized path difference introduced by a linear diffractive grating, for any orientation of the grating with respect to the irradiation direction and at collection directions for which diffraction is observed. Coatings with diffraction pigments include all possible orientations, and, analogously to Eq. 5, we can express the path difference introduced by the diffraction pigments embedded in the coating as:

$$
\Delta G \approx \frac{d}{n} \Phi\left(\theta_{\mathrm{i}}, \theta_{\mathrm{r}}, \phi_{\mathrm{r}}\right)
$$

The spectral reflectance of the coatings with diffraction pigments at a given geometry is mainly determined by the path differences introduced by it, taking relative maximum values for:

$$
\Delta G=\lambda z, \quad z= \pm 1, \pm 2, \ldots
$$

where $\lambda$ is the wavelength of incident radiation. Therefore, the condition for the highest contribution of the diffraction pigment to the total BRDF is:

$$
\frac{d \Phi\left(\theta_{\mathrm{i}}, \theta_{\mathrm{r}}, \phi_{\mathrm{r}}\right)}{n \lambda} \approx \pm 1, \pm 2, \ldots
$$

Therefore, the geometric factor $\Phi\left(\theta_{\mathrm{i}}, \theta_{\mathrm{r}}, \phi_{\mathrm{r}}\right)$ in Eq. 11 may be used to predict the component of the spectral distribution of the coating's BRDF exclusively due to diffraction:

$$
f_{\mathrm{r}, \mathrm{d}}\left(\theta_{\mathrm{i}}, \theta_{\mathrm{r}}, \phi_{\mathrm{r}} ; \lambda\right)=f(\Phi, \lambda)
$$

For a given geometry, the relative maxima at different orders $z= \pm 1, \pm 2, \ldots$ are obtained for specific wavelengths. If we make the approximation that the value of the partial BRDF at the different orders are much larger than out of those orders, we can write:

$$
f_{\mathrm{r}, \mathrm{d}}\left(\theta_{\mathrm{i}}, \theta_{\mathrm{r}}, \phi_{\mathrm{r}} ; \lambda\right)=\sum_{k=-\left|z_{\max }\right|}^{z_{\max }} \Lambda_{\mathrm{k}}(\lambda) \Theta_{\mathrm{k}}(\Phi)
$$

where $k$ is the order index, $\Lambda_{\mathrm{k}}$ accounts for the spectral dependence of reflectance for order $k$, and $\Theta_{\mathrm{k}}(\Phi)$ for the geometrical dependence of reflectance for order $k$.

The spectral BRDF of coatings with diffraction pigments can be understood as a linear combination of several reflection terms referred to different mechanisms, one of them being the diffraction mechanism due to diffraction pigments. So, we can write the complete spectral BRDF of coatings with diffraction pigments as:

$$
f_{\mathrm{r}, \mathrm{c}}\left(\theta_{\mathrm{i}}, \theta_{\mathrm{r}}, \phi_{\mathrm{r}} ; \lambda\right)=f_{\mathrm{r}, 0}\left(\theta_{\mathrm{i}}, \theta_{\mathrm{r}}, \phi_{\mathrm{r}} ; \lambda\right)+\sum_{k=-\left|z_{\max }\right|}^{z_{\max }} \Lambda_{\mathrm{k}}(\lambda) \Theta_{\mathrm{k}}(\Phi)
$$

where $f_{\mathrm{r}, 0}$ is the component of the spectral BRDF independent of diffraction on pigments.

In previous works, we have used a Principal Components Analysis (PCA, see Appendix) approach [14, 15] to express the spectral BRDF of coatings with interference pigments as:

$$
f_{\mathrm{r}}\left(\theta_{\mathrm{i}}, \theta_{\mathrm{s}}, \phi_{\mathrm{s}} ; \lambda\right)=\left\langle f_{\mathrm{r}}\left(\theta_{\mathrm{i}}, \theta_{\mathrm{s}}, \phi_{\mathrm{s}}\right)\right\rangle_{\lambda}\left[1+\sum_{j=1}^{S} c_{j}\left(\theta_{\mathrm{i}}, \theta_{\mathrm{s}}, \phi_{\mathrm{s}}\right) H_{j}(\lambda)\right]
$$

This equation corresponds to the multiplication of two factors: the spectral average of $f_{\mathrm{r}}\left(\left\langle f_{\mathrm{r}}\right\rangle_{\lambda}\right)$ with just geometrical dependence, and the sum of a number of components (within square brackets), being the first one the unity. Every addend is factorized in two: One factor carrying the spectral information, $H_{j}(\lambda)$ (with an average value of 0 and a standard deviation value of 1 ), and another factor carrying the geometrical information, $c_{j}\left(\theta_{\mathrm{i}}, \theta_{\mathrm{s}}, \phi_{\mathrm{s}}\right)$, that can be regarded as the weighting coefficients of every spectral distribution at the different geometrical configurations. Since theoretical Eq. 17 can be expressed in a similar way than Eq. 18, we have applied this PCA procedure to the spectral BRDF data of coatings with diffraction pigments.

\section{CHARACTERIZATION OF COATINGS}

The spectral BRDFs shown in this article were experimentally obtained with the goniospectrophotometer GEFE, designed and developed at IO-CSIC $[6,7]$. GEFE comprises three systems (see Fig. 4): the irradiation system, the positioning system and the detection system. The first one is fixed, whereas the other two systems are mobile: The sample is placed with the required orientation relative to the incoming beam, while the detector is attached to a cogwheel so as to be able to revolve around the sample. This arrangement permits a fast and accurate sampling. The design allows different types of light sources to be used, and in this case we used a wide-band xenon lamp. In order to irradiate uniformly and with a collimated beam the specimens, a Köhler optical system is used. Both irradiation and collection are directional, within a half-angle of less than $1^{\circ}$. The irradiation on the sample can be considered as unpolarized light.

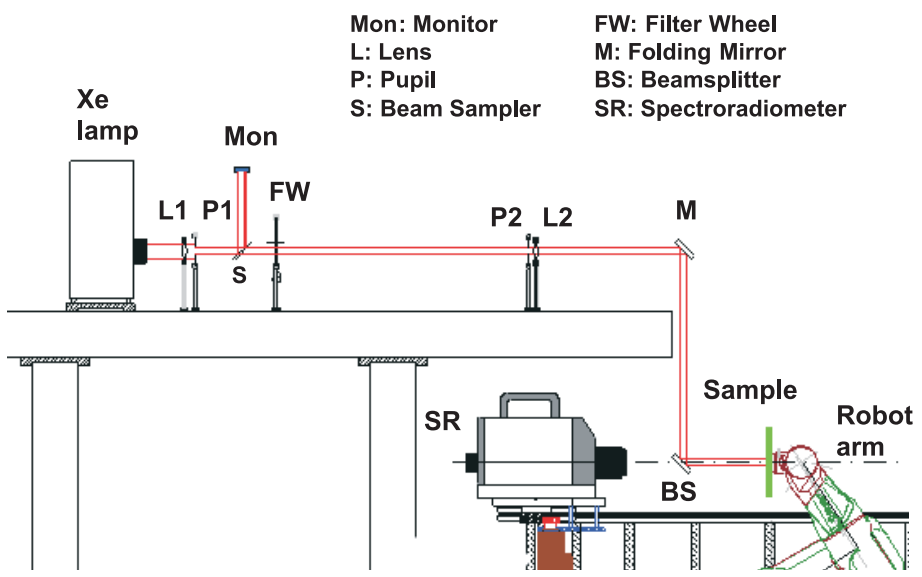

Fig. 4. Sketch of the goniospectrophotometer GEFE.

The spectral BRDF of six coatings with SpectraFlair ${ }^{\circledR}$ diffraction pigments (Silver 1500-14, Silver 1500-20, Silver 1500-35, Plus 25, Bright Silver 20, Bright Silver HSA-20) [16] was measured. These pigments are linear diffractive gratings with grating constant $g=1 / d=1500 \mathrm{~mm}^{-1}$. The three pigments of the 1500 series correspond to different average particle sizes (14 $\mu \mathrm{m}, 20 \mu \mathrm{m}$ and $35 \mu \mathrm{m})$ and different grades of visual texture 
(fine, standard and coarse). Plus 25 has an average particle size of $23.5 \mu \mathrm{m}$, and three times more specific surface area (surface area per weight unit of the pigment). On the other hand, Bright Silver 20 pigments offer dramatic, diamond like sparkle usually associated with much larger particle-size pigments. The thinner Bright Silver HSA offers a more liquid silver appearance, with three times more specific surface area.

In this work, the spectral BRDF was measured for the geometries resulting from the combination of the following spherical coordinates: eight polar angles for both irradiation and collection directions (from $\theta_{\mathrm{i}}$ and $\theta_{\mathrm{r}}=0^{\circ}$ to $70^{\circ}$, with angular steps of $\left.10^{\circ}\right)$, and two azimuth angles for collection directions $\left(\phi_{\mathrm{r}}=0^{\circ}\right.$ and $180^{\circ}$, within the incidence plane), except for the sample Silver 1500-14, for which additional out-of-plane geometries were studied (from $\phi_{\mathrm{r}}=0^{\circ}$ to $180^{\circ}$, with angular steps of $30^{\circ}$ ). We assumed invariance with respect to the azimuth angle of irradiation and we kept it constant at zero degrees (azimuth origin). Notice that measurements are said to be within the incidence plane (in-plane) when the azimuth angle of the irradiation is $0^{\circ}$ or $180^{\circ}$, and out-of-plane otherwise.

\section{RESULTS}

The spectral BRDF measurements already show that colors of coatings Silver 1500-14, Silver 1500-20, Silver 1500-35 and Plus 25 change similarly with the geometry (although not in the same extent), and that coatings Bright Silver and Bright Silver HSA have also a similar behavior between them, with a lower color shift. Therefore, we will discuss the results of Silver 1500-14 and Bright Silver HSA as representative coatings. For these two coatings, the spectral functions or eigenspectra $H_{j}(\lambda)$ (Eq. 18), corresponding with the four highest eigenvalues, are shown in Fig. 5. For the coating Silver 1500-14, the components corresponding with the two first eigenvalues $\left(H_{1}\right.$ and $\mathrm{H}_{2}$ ) represent $86 \%$ of the total data variance, the four first represent $97 \%$ and the six first represent $99 \%$, whereas, for the case of Bright Silver HSA, they represent $99.5 \%$, $99.9 \%$ and $99.95 \%$, respectively. So, it is possible to use a reduced
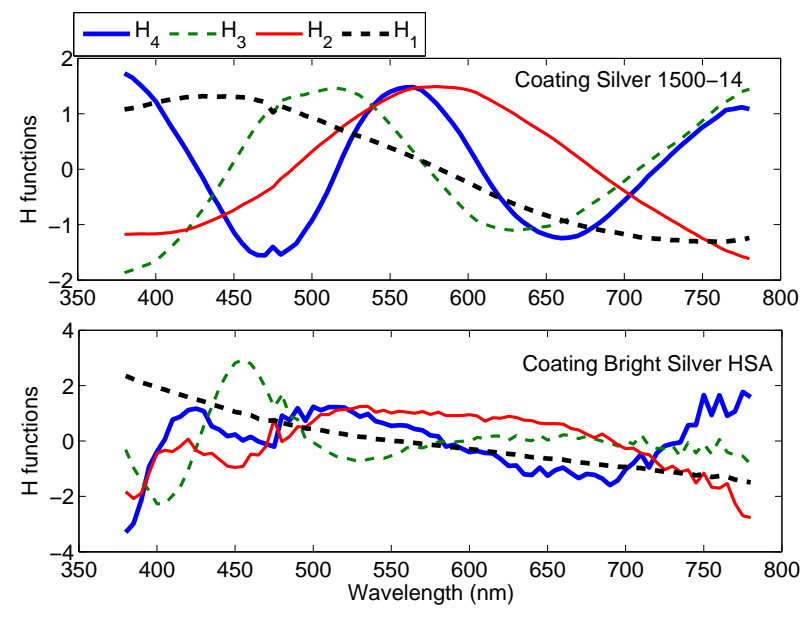

Fig. 5. Four most relevant eigenspectra $H_{j}(\lambda)$ (highest eigenvalues of spectral) BRDFs of Silver 1500-14 (upper) and Bright Silver HSA (lower).

set of spectral distributions to describe the spectral BRDF of these samples. On the other hand, weighting coefficients, $c_{j}$ (

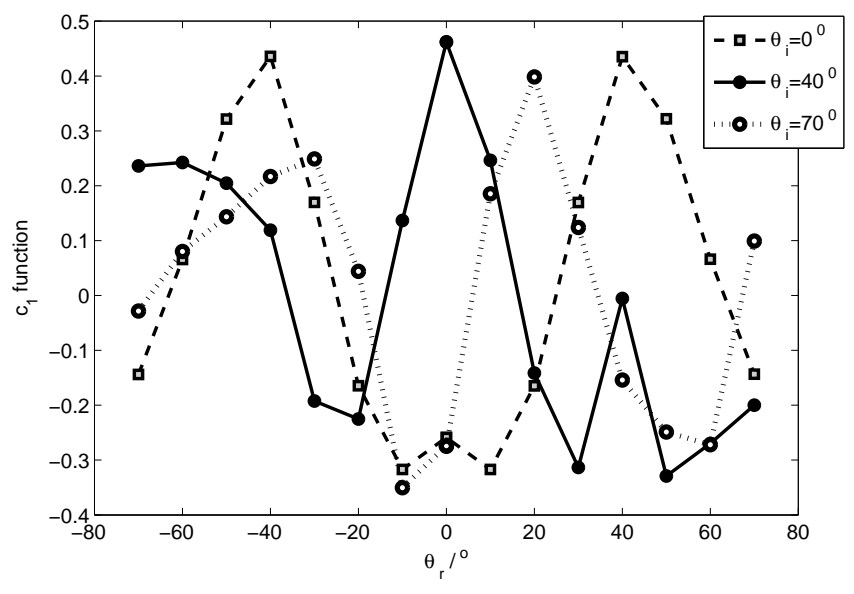

Fig. 6. Dependence of $c_{1}$-values on spherical coordinates (Coating Silver 1500-14). In this figure $\theta_{\mathrm{r}}$ is negative if the collection direction is contained in the same half-plane than the specular direction.

(Eq. 18)), vary in a great extent for different pairs of irradiation and collection directions. As an example, $c_{1}$ of Silver 1500-14 for three irradiation angles is plotted as a function of the polar angle of the collection direction in Fig. 6. We can see that the variation of this weighting coefficients with respect to the polar angle is hard to be predicted, since its value varies also with the polar angle of irradiation. Is it possible to use another variable considering geometrical specifications for both irradiation and collection directions? The basic approach would be to use the aspecular angle $\left(\theta_{\text {asp }}\right)$ as geometric variable, defined as the angular deviation of collection direction from specular direction. Since this is related with the optical path difference, the reflected diffraction pattern should be kept almost constant with this angle. By representing $c_{1}$ as a function of $\theta_{\text {asp }}$ a more predictable behavior, almost independent of $\theta_{i}$ is found (see the top plot in Fig. 7, for $c_{1}$ of Silver 1500-14, where only data of the in-plane geometries were used). Nevertheless, the eigenvector $c_{1}$ still has got a considerable variation at the same $\theta_{\text {asp }}$. Notice that the values of $c_{1}$ for all in-plane geometries studied are represented in this plot, whereas only those corresponding with geometries at irradiation polar angles $\theta_{\mathrm{i}}=0^{\circ}, 40^{\circ}$ and $70^{\circ}$ are shown in Fig. 6.

If $c_{1}$ is represented as a function of the geometric factor $\Phi\left(\theta_{\mathrm{i}}, \theta_{\mathrm{r}}, \phi_{\mathrm{r}}\right)$ defined in Eq. 11, a much clear periodic structure, almost independent of the polar angle, is found (see the bottom plot in Fig. 7). Then, if we were able to characterize the dependence of $c_{1}$ with respect to $\Phi$, we could estimate the contribution of the eigenspectra $H_{1}$ to the spectral BRDF for a given geometry. But this would not mean that the spectral BRDF can be completely estimated. For that, we would need to be able to estimate the contribution of other components. And it can be done because, as shown in Fig. 8 for geometries within incidence plane $\left(\phi_{\mathrm{r}}=0^{\circ}\right.$ and $\left.180^{\circ}\right)$, the $c_{j}$-values of the most relevant components have also a smooth dependence on $\Phi$ (shown for Silver 1500-14 and Bright Silver HSA). This is also the case for out-of-plane geometries $\left(\phi_{\mathrm{r}}=30^{\circ}, 60^{\circ}, 90^{\circ}, 120^{\circ}\right.$ and $\left.150^{\circ}\right)$, as shown in Fig. 9 for Silver 1500-14. We must notice that lower values of $\Phi$ correspond with directions closer to the specular direction, with $\phi_{\mathrm{r}}=180^{\circ}$. 

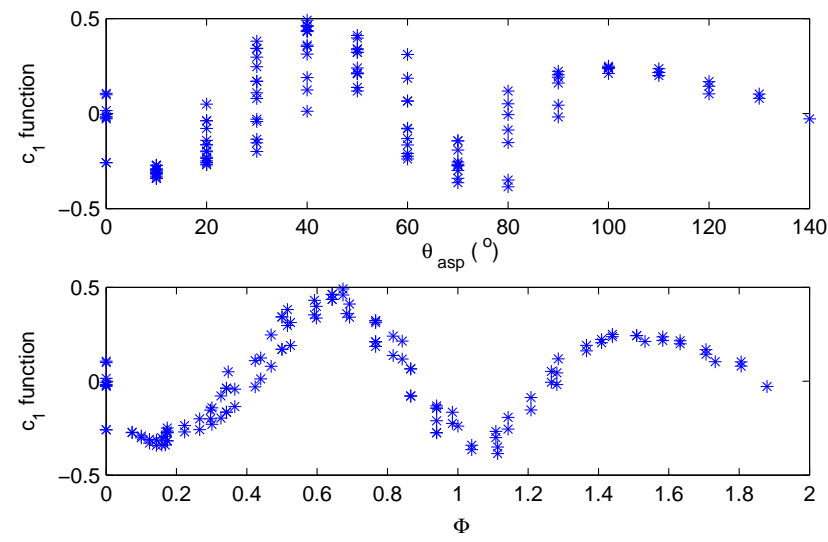

Fig. 7. Dependence of $c_{1}$-values on the aspecular angle (top), and on the geometric factor defined in Eq. 11 (bottom). (Coating Silver 1500-14).
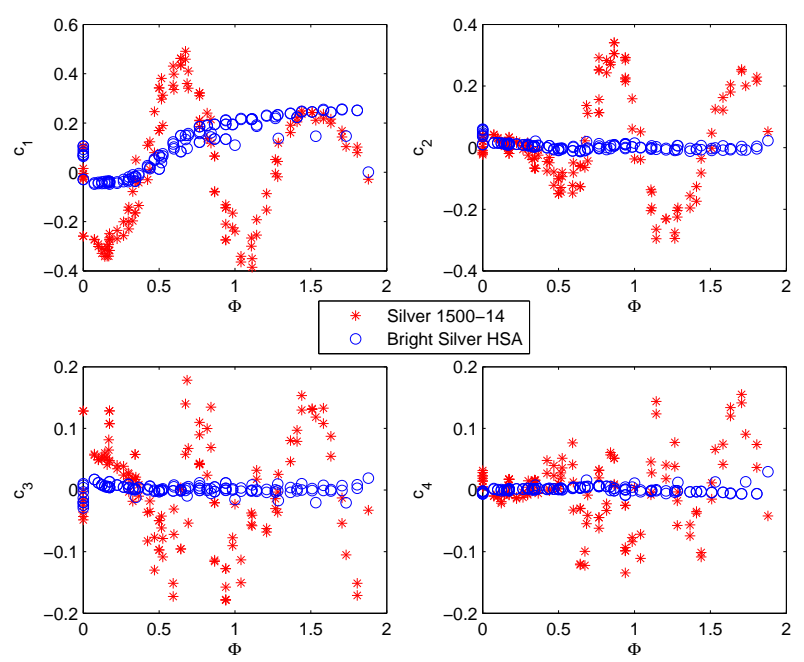

Fig. 8. Variation with $\Phi$ of $c_{j}$ values corresponding with the four highest eigenvalues of spectral BRDFs of Silver 1500-14 and Bright Silver HSA, for $\phi_{\mathrm{r}}=0^{\circ}$ and $180^{\circ}$ ).
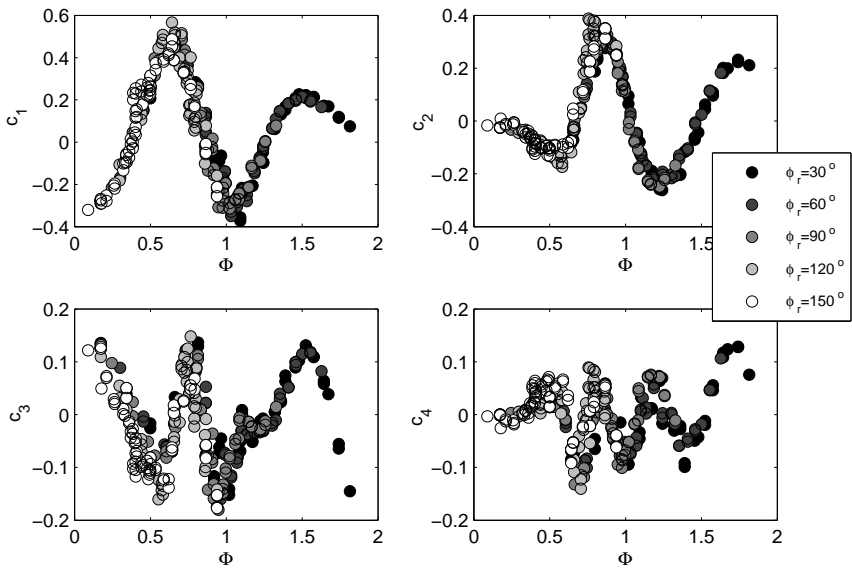

Fig. 9. Variation with $\Phi$ of the $c_{j}$ values corresponding with the four highest eigenvalues of spectral BRDFs of Silver 150014 , for $\phi_{\mathrm{r}}=30^{\circ}, 60^{\circ}, 90^{\circ}, 120^{\circ}$ and $150^{\circ}$.

In addition, the spectrally-averaged $\operatorname{BRDF}\left(\left\langle f_{\mathrm{r}}\right\rangle_{\lambda}\right)$ presents a very predictable behaviour when expressed as a function of $\Phi$, as it is shown in Fig. 10 for all the studied coatings. The values of $\left\langle f_{\mathrm{r}}\right\rangle_{\lambda}$ for coatings Silver 1500-14, Silver 1500-20, Silver 150035 and Plus 25 are almost identical (bottom plot), and the same can be said for Bright Silver and Bright Silver HSA (top plot). Again, this is so also for out-of-plane geometries, as shown in Fig. 11 for Silver 1500-14 as an example.
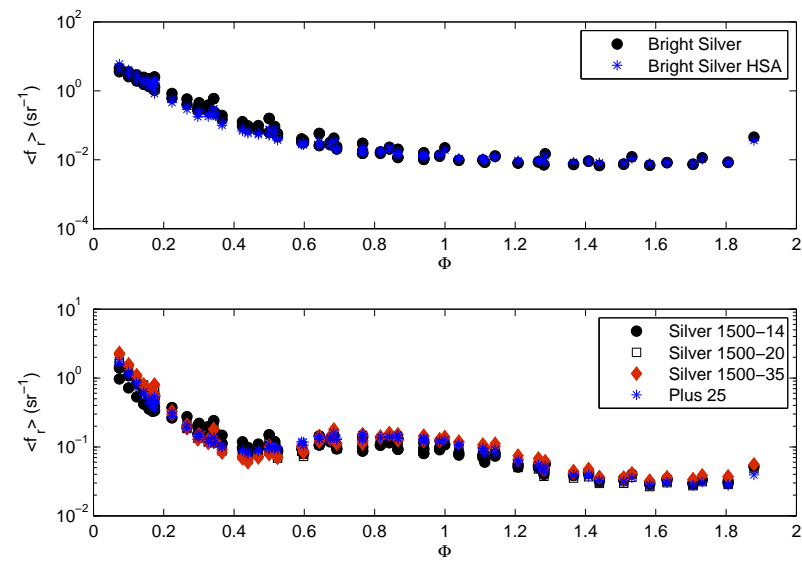

Fig. 10. Variation with $\Phi$ of the spectrally-averaged BRDF $\left(\left\langle f_{\mathrm{r}}\right\rangle_{\lambda}\right)$ of coatings Silver 1500-14, Silver 1500-20, Silver 1500-35 and Plus 25 (bottom plot) and Bright Silver and Bright Silver HSA (top plot).

The main difference between the coatings Silver 1500-14, Silver 1500-20 and Silver 1500-35 is the size of the diffraction pigments $(14 \mu \mathrm{m}, 20 \mu \mathrm{m}$ and $35 \mu \mathrm{m}$, respectively). It produces that the contribution of the diffraction to the total spectral BRDF is different for them. As an example, the variation of the first weighting coefficient, $c_{1}$, for these three coatings is shown in Fig. 12. This coefficient, which represents the spectral contribution of the eigenspectrum contributing the most to the variance, has a periodic structure with a wider amplitude as larger is the size of the diffraction pigments in the coatings. 


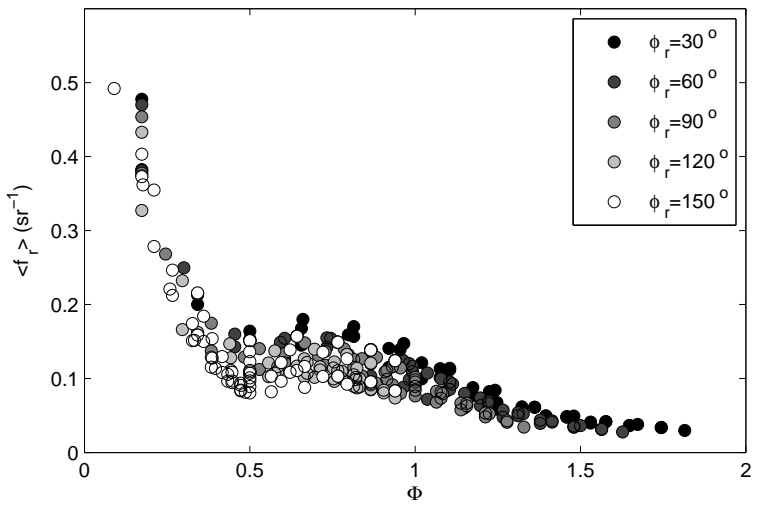

Fig. 11. Variation with $\Phi$ of the spectrally-averaged BRDF $\left(\left\langle f_{\mathrm{r}}\right\rangle_{\lambda}\right)$ of coating Silver 1500-14, for out-of-plane measurements.

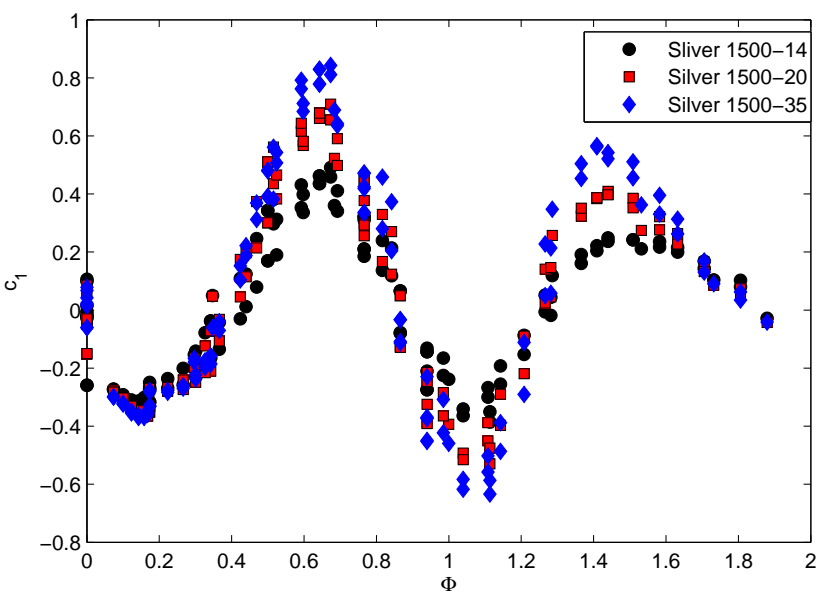

Fig. 12. Variation with $\Phi$ of the first weighting coefficient, $c_{1}$, of coatings Silver 1500-14, Silver 1500-20 and Silver 1500-35, having different diffraction pigment sizes $(14 \mu \mathrm{m}, 20 \mu \mathrm{m}$ and $35 \mu m$, respectively).

\section{ANALYSIS OF CHROMATIC COORDINATES}

If we calculate CIELAB chroma $\left(C_{\mathrm{ab}}^{*}\right)$ and hue angle $\left(h_{\mathrm{ab}}^{*}\right)$ from the BRDF measurements of the coating Silver 1500-14, using the CIE-D65 illuminant and the CIE-1964 standard observer, and plot them as a function of the polar angle of the collection direction for different incidence angles, graphs shown in Fig. 13 are obtained. As seen before for the spectral BRDF-based analysis, no clear relation can be established between them and $\theta_{\mathrm{r}}$. If we apply the same rationale proposed before to these visual attributes (CIELAB chroma and hue angle), and we plot them as shown in Figs. 14 (chroma) and 15 (hue angle), a relation of these visual attributes can be foreseen. Therefore, we conclude that $\Phi$ is a relevant geometric variable to study the spectral reflectance and color variations of coatings with diffraction pigments, more relevant than the aspecular angle.
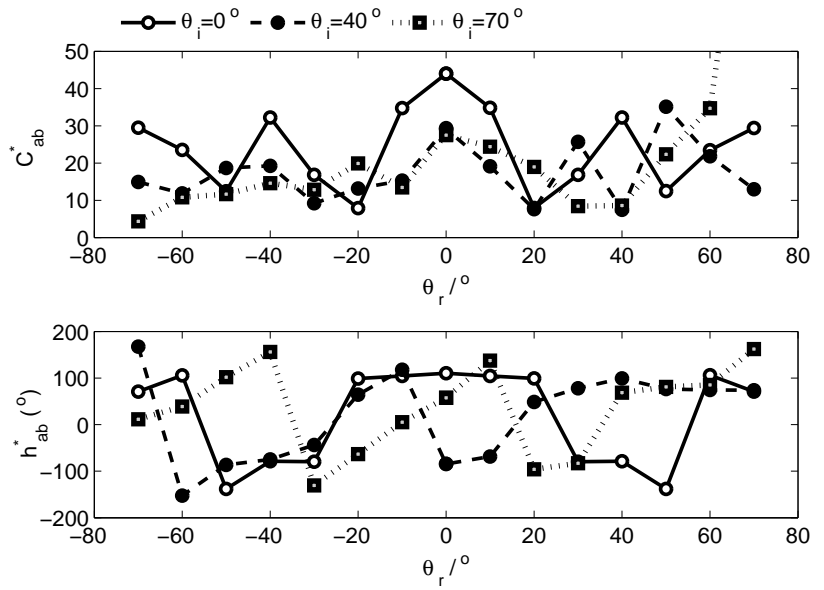

Fig. 13. Variation of CIELAB chroma (top) and CIELAB hue angle (bottom) with the polar angle of the collection direction for different incidence angles (Coating Silver 1500-14).
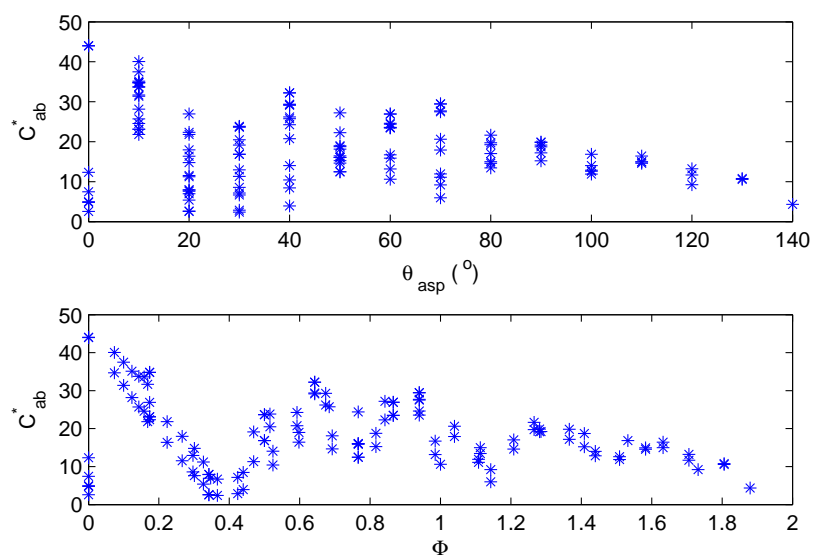

Fig. 14. Comparison of the variation of CIELAB chroma with the aspecular angle (top) and the geometric factor $\Phi$ (bottom). (Coating Silver 1500-14).

The main advantage of applying the PCA-based approach on the spectral BRDF is that it allows the color at a different illumination or geometrical condition to be calculated, and not only at the conditions of characterization (such as illuminant 

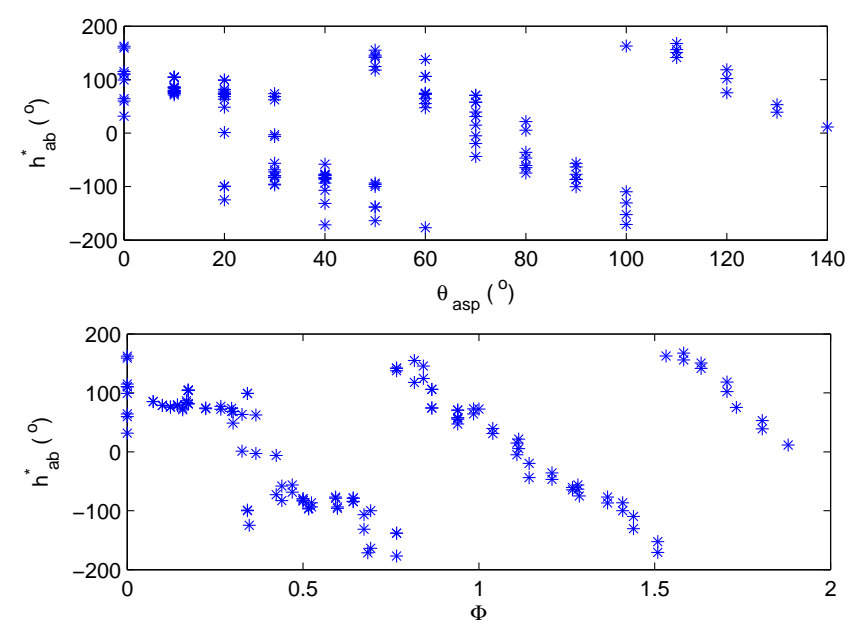

Fig. 15. Comparison of the variation of CIELAB hue angle with the aspecular angle (top) and the geometric factor $\Phi$ (bottom). (Coating Silver 1500-14).

spectral distribution or definition of irradiation/collection solid angles), whereas the chromatic approach is only valid at those specific conditions of characterization.

The color of the coating Silver 1500-14 for different geometries is represented in Fig. 16. Every plot within it represents a different incidence direction and the colors, encoded by sRGB color space, are arranged in a matrix according to $\theta_{\mathrm{r}}$ and $\phi_{\mathrm{r}}$ spherical coordinates. White color represents saturation, which is obtained mainly around specular directions. This figure allows the complex chromaticity of these coatings to be visually evaluated.
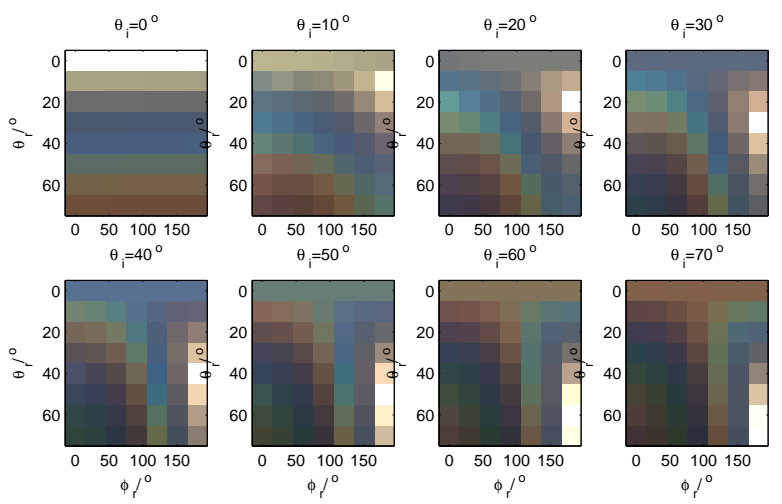

Fig. 16. Representation of the color gamut of the coating Silver 1500-14, arranged in a matrix defined by spherical coordinates of collection direction. Every plot corresponds to a different irradiation direction

A different arrangement is shown in Fig. 17, where the same colors are located now from lower to larger geometric factor $\Phi$, proving that this variable is the most relevant for the chromatic description of coatings with diffraction pigments.

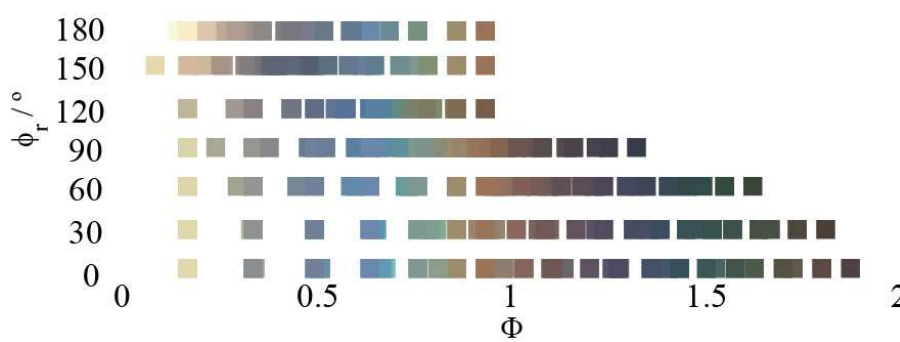

Fig. 17. Chromatic representation of the coating Silver 150014 for different geometries, arranged by the geometric factor $\Phi$. In order to avoid superposition of colors, seven rows are displayed, one for a fixed $\phi_{\mathrm{r}}$.

\section{COMPARISON BETWEEN COATINGS WITH IN- TERFERENCE PIGMENTS AND COATINGS WITH DIFFRACTION PIGMENTS}

Although both kinds of coatings are regarded as iridescent or goniochromatic, these coatings present two important differences which need to be taken into account for their BRDF/color characterization. Firstly, whereas the special effect produced by the interference pigments mainly depends on the incidence angle on them $\left(\theta_{\text {inc }}\right)[15,17]$, for coatings with diffraction pigments this dependence lies on the geometric factor $\Phi$, as has been shown in this work, or less accurately on the aspecular angle. To support the discussion on chromatic differences, we have represented in CIE $\mathrm{a}^{*}, \mathrm{~b}^{*}$-diagrams the CIELAB chromatic coordinates of a coating with interference pigments (Cyan Purple $230 \mathrm{~L}$ ) and those of a coating with diffraction pigments (Spectraflair Silver 1500-14) (Figs. 18.a and 18.b, respectively). The coordinates were calculated with illuminant CIE D65 and standard observer CIE 1964. The widely-used interference lines (the loci of calculated color coordinates for geometries with a fixed aspecular angle but different incidence angles) are shown. In diagram (a) (pigment Cyan Purple 230L), by moving along the length of the interference lines, the complete hue angle $\left[h_{\mathrm{ab}}^{*}=\arctan \left(a^{*} / b^{*}\right)\right]$ variation is obtained, and that is because the average $\theta_{\text {inc }}$ on the pigments changes at different incidence angles. Something completely different is observed in diagram (b) (pigment Spectraflair Silver 1500-14), where the hue angle variation for a given interference line does not give insight into the total hue angle variation of the coating.

The second important difference is based on the fact that the special effect produced by interference pigments can only be observed at specular angles with respect to them, whereas the effect of the diffraction pigments takes place at different angles. As a consequence, unlike the coatings with diffraction pigments, the coatings with interference pigments show the hue angle variation mainly at low specular angles, since the pigments are almost (but not perfectly) parallel to the coating surface. Their effect on the color is better observed in Fig. 18.a: Interference lines with lower aspecular angles have got a higher chroma $\left[C_{\mathrm{ab}}^{*}=\sqrt{a^{* 2}+b^{* 2}}\right]$ and a wider hue angle shift (as determined by the interference pigments), whereas, for the highest aspecular angles, chroma and hue angle variations are smaller (as provided by the conventional absorption pigments). This is not observed in Fig. 18.b, where the chroma does not seem to be correlated to the aspecular angle. In this case, the aspecular angle seems to be more correlated to the hue angle, as expected from our result: the relative spectral distribution of the BRDF mainly depends on the geometric factor $\Phi$, which has 


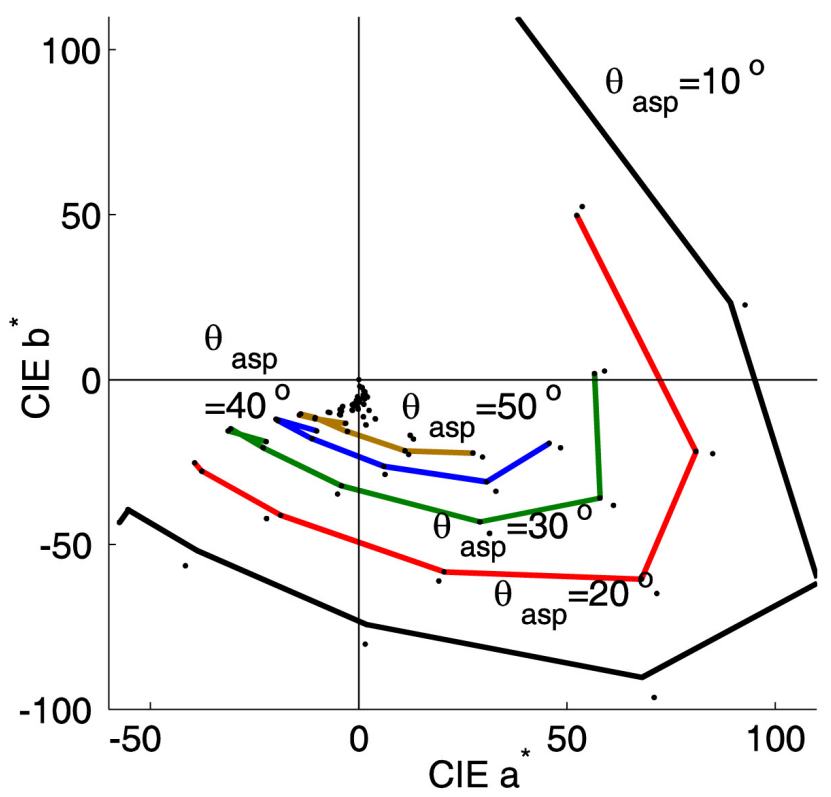

(a) Coating with interference pigments: Cyan Purple $230 \mathrm{~L}$

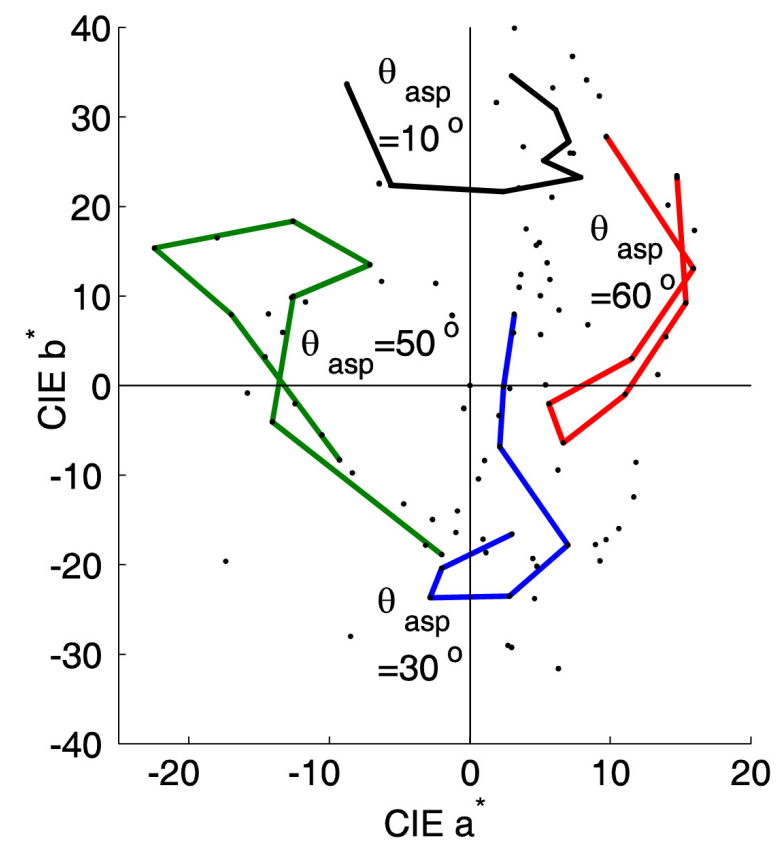

(b) Coating with diffraction pigments: Sprectraflair Silver 1500-14.

Fig. 18. CIE $a^{*}, b^{*}$-diagrams for one coating with interference pigments (a) and one with diffraction pigments (b). some correlation to the aspecular angle $\left(\theta_{\text {asp }}\right)$.

The black dots in Fig. 18 represent measures at other geometries. Dots in diagram (b) have a more chaotic distribution than those in (a). We may conclude that, although the color gamut of coatings with interference pigments can be somehow characterized by looking at color coordinates, it is definitively necessary to use a spectral BRDF-based characterization to foresee the color gamut of coatings with diffraction pigments.

Finally, our measurements and analysis reveal that commercially-available portable goniospectrophotometers are not adequate to characterize coatings with diffraction pigments, which demand a higher number of aspecular angles not available at those instruments. The result shown in Fig. 8 suggests that the characterization might be done with a single incidence angle, although this would need to be verified in studies with a larger set of samples.

\section{CONCLUSIONS}

The spectral Bidirectional Reflectance Distribution Function (BRDF) of six coatings with diffraction SpectraFlair pigments has been measured using the robot-arm-based goniospectrophotometerer GEFE. A PCA-based procedure has been used to study the BRDF measurements. From both a physical model and experimental data evaluation, we have defined a geometric factor related to the optical path difference, which may be considered the relevant geometric variable to describe the spectral reflectance and color gamut of coatings with diffraction pigments. We have compared and discussed the color shifts of coatings with interference pigments and with diffraction pigments. We have concluded that, although the color gamut of coatings with interference pigments can be somehow characterized by looking at color coordinates, it is definitively necessary to use a spectral BRDF-based characterization to foresee coatings with diffraction pigments. Our measurements and analysis have revealed that more aspecular measurement angles has to be added to commercially-available portable goniospectrophotometers to characterize the color gamut of goniochromatic coatings based on diffraction pigments.

\section{APPENDIX: PRINCIPAL COMPONENTS ANALYSIS ON SPECTRAL BRDF}

The Principal Components Analysis is a powerful statistical technique capable of identifying and quantifying orthogonal contributions to the total variance of a collection of data. The key element is the definition of a multidimensional random variable $F=\left\{F_{1}, F_{2}, \ldots, F_{N}\right\}$, having $M$ realizations of it. For the BRDF data, this variable is made up of a concatenation of $N F_{i}$ spectra, each one corresponding to the measured spectral $\mathrm{BRDF}$ for a given angular configuration. The set of values extracted from the different spectra corresponding to a particular wavelength is taken as a particular realization of the multidimensional variable; then, the number of realizations, $M$, coincides with the number of wavelengths. The collection of data is arranged as a matrix having $N$ columns and $M$ rows. Each row contains the BRDF for every angular configuration at a specific wavelength. The covariance matrix of the spectra is obtained as an $N \times N$ matrix, $\mathbf{S}_{F}$. This covariance matrix is typically non diagonal, thus revealing the inner correlations between the spectra. The PCA method diagonalizes this covariance matrix and produces three types of elements: $N$ eigenvalues, $\gamma_{j}, N$ eigenvectors, $e_{j}$, and $N$ eigenspectra, $A_{j}$. The eigenvalues are 
arranged in decreasing order. They quantify the importance and the contribution to the total variance of the data of their associated eigenspectra. Actually, $\gamma_{j}$ is the variance of the eigenspectra $A_{j}$. The eigenvectors can be seen as the coefficients of the transformation from the correlated variables given by the spectra to a new set of uncorrelated variables expressed by the eigenspectra. These eigenvectors can be arranged as an $N \times N$ orthogonal matrix, $\mathbf{E}^{T}=\mathbf{E}^{-1}$ ( ${ }^{T}$ means transposition). Finally, the eigenspectra, $A_{j}$, describe uncorrelated data that provide spectral insight about the different contributions to the BRDF. $A_{j}$ is calculated as:

$$
A_{j}=\sum_{i=1}^{N} e_{j i} \bar{F}_{i}
$$

where $\bar{F}_{i}$ is a null-mean spectrum obtained from the original $i$ th spectrum, $F_{i}$, for instance, by subtraction. In our case, we scaled this variable as:

$$
\bar{F}_{i}=\frac{F_{i}}{\left\langle F_{i}\right\rangle}-1
$$

The importance of a given eigenspectrum, $A_{j}$, within the data set is quantified by its associated eigenvalue, $\gamma_{j}$. By using the eigenvectors it is possible to migrate from the experimental coordinate system, $\left\{F_{1}, \ldots, F_{N}\right\}$, to the eigenspectra coordinate system, $\left\{A_{1}, \ldots, A_{N}\right\}$, and vice versa. This transformation can be written as follows:

$$
\bar{F}_{i}=\sum_{j=1}^{N} e_{i j} A_{j}
$$

From this equation we may obtain a filtered version of the spectra by selecting a customized subset of eigenvectors and their associated eigenspectra. This is easily done by choosing a subset of subscripts within the sum. Then, it is also possible to remove or to select a given collection of contributions characterized by their associated eigenspectra. Notice that Eq. 18 is obtained form Eq. 21.

\section{FUNDING INFORMATION}

This work was done within the EMRP IND52 Project "Multidimensional reflectometry for industry" (xD-Reflect). The EMRP is jointly funded by the EMRP participating countries within EURAMET and the European Union. Authors are also grateful to Comunidad de Madrid for funding the project SINFOTONCM: S2013/MIT-2790, and the Spanish Ministry of Economy and Competitiveness for funding the project DIP2015-65814.

\section{REFERENCES}

1. H.-J. Streitberger and K.-F. Dossel, Automotive paints and coatings (Wiley-Vch, 2008).

2. S. Berthier, Iridescences: the physical colors of insects (Springer Science \& Business Media, 2007).

3. B. D. Wilts, K. Michielsen, H. De Raedt, and D. G. Stavenga, "Iridescence and spectral filtering of the gyroid-type photonic crystals in parides sesostris wing scales," Interface Focus p. rsfs20110082 (2011).

4. P. Vukusic and J. R. Sambles, "Photonic structures in biology," Nature 424, 852-855 (2003).

5. F. E. Nicodemus, J. C. Richmond, and J. J. Hsia, Geometrical considerations and nomenclature for reflectance (Natl. Bur. Stand. Monogr. 160, 1977).
6. A. M. Rabal, A. Ferrero, J. Campos, J. L. Fontecha, A. Pons, A. M. Rubiño, and A. Corróns, "Automatic gonio-spectrophotometer for the absolute measurement of the spectral brdf at in-and out-of-plane and retroreflection geometries," Metrologia 49, 213-223 (2012).

7. B. Bernad, A. Ferrero, A. Pons, M. L. Hernanz, and J. Campos, "Upgrade of goniospectrophtometer GEFE for near-field scattering and fluorescence radiance measurements," in "IS\&T/SPIE Electronic Imaging," (International Society for Optics and Photonics, 2015), pp. 93980E-93980E.

8. A. Ferrero, E. Perales, A. M. Rabal, J. Campos, F. M. Martínez-Verdú, E. Chorro, and A. Pons, "Color representation and interpretation of special effect coatings," Journal of the Optical Society of America A 31, 436-447 (2014)

9. E. Kirchner and A. Ferrero, "Isochromatic lines as extension of the Helmholtz reciprocity principle for effect paints," Journal of the Optical Society of America A 31, 1861-1867 (2014).

10. A. Ferrero, J. Campos, E. Perales, F. M. Martínez-Verdú, I. van der Lans, and E. Kirchner, "Global color estimation of special-effect coatings from measurements by commercially available portable multiangle spectrophotometers," Journal of the Optical Society of America A 32, 1-11 (2015)

11. G. A. Klein, Industrial color physics, vol. 154 (Springer, 2010).

12. W. R. Cramer and F. J. Maile, "Rainbows made to order," European Coatings Journal 16, 52-56 (2016).

13. N. Rogelj, I. Poberaj, and M. K. Gunde, "Goniospectrophotometric space curves of diffraction gratings and their applicability as appearance fingerprints," Applied optics 52, 8355-8362 (2013).

14. A. Ferrero, A. M. Rabal, J. Campos, A. Pons, and M. L. Hernanz, "Variables separation of the spectral BRDF for better understanding color variation in special effect pigment coatings," Journal of the Optical Society of America A 29, 842-847 (2012).

15. A. Ferrero, A. M. Rabal, J. Campos, F. Martínez-Verdú, E. Chorro, E. Perales, A. Pons, and M. L. Hernanz, "Spectral BRDF-based determination of proper measurement geometries to characterize color shift of special effect coatings," Journal of the Optical Society of America A 30, 206-214 (2013)

16. J. U. Corporation, "Spectraflair pigments: Bringing form to life," http://www.viavisolutions.com/sites/default/files/technical-libraryitems/spectraflair-ds-ccs-ae.pdf (2013). [Online; accessed 31-May2016].

17. E. Kirchner and W. Cramer, "Making sense of measurement geometries for multi-angle spectrophotometers," Color Research \& Application 37, 186-198 (2012). 